\title{
Quantum Yang-Mills on a Riemann Surface
}

\author{
Dana S. Fine \\ Department of Mathematics, The University of Massachusetts at Dartmouth, North Dartmouth, \\ MA 02747, USA
}

Received December 10, 1990; in revised form March 14, 1991

\begin{abstract}
We obtain the quantum expectations of gauge-invariant functions of the connection on a $G=S U(N)$ product bundle over a Riemann surface of genus $g$. We show that the space $\mathscr{A} / \mathscr{G}_{m}$ of connections modulo those gauge transformations which are the identity at one point is itself a principal bundle with affine linear fiber. The base space $\mathrm{Path}^{2 g} G$ consists of $2 g$-tuples of paths in $G$ subject to a relation on their endpoint values. Quantum expectations are iterated path integrals over first the fiber then over Path ${ }^{2 g} G$, each with respect to the push-forward to $\mathscr{A} / \mathscr{G}_{m}$ of the measure $e^{-S(A)} \mathscr{D} A$. Here, $S(A)$ denotes the Yang-Mills action on $\mathscr{A}$. We exhibit a global section of $\mathscr{A} / \mathscr{G}_{m}$ to define a choice of origin in each fiber, relative to which the measure on the fiber is Gaussian. The induced measure on Path $^{2 g} G$ is the product of Wiener measures on the component paths, conditioned to preserve the endpoint relation. Conformal transformations of the metric on $M$ act by reparametrizing these paths. We explicitly compute the partition function in the general case and the expectations of functions of certain products of Wilson loops in the case $g=1$.
\end{abstract}

\section{Introduction}

In [2], we treated Yang-Mills on $S^{2}$, deriving the quantum expectation of a gauge-invariant function of the connection. To do so, we interpreted the path intergal as an integral with respect to a measure $\mu$ on $\mathscr{A} / \mathscr{G}_{m}$, the space of connections modulo gauge transformations which are the identity at a point $m$. We showed that $\mathscr{A} / \mathscr{G}_{m}$ fibers over $\Omega G$, based loops in the symmetry group, and we formally decomposed $\mu$ into a measure on the fiber and a measure on the base.

Sengupta [5] treats the same problem from the perspective of stochastic parallel transports, as developed for Yang-Mills on $R^{2}$ in Gross, King and Sengupta [4]. His results and those of [2] agree where they overlap. In a future paper, we intend to check for further agreement.

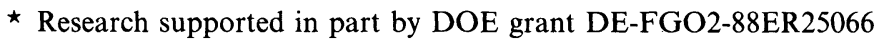


In this paper, we extend the techniques of [2] to describe the quantum theory of Yang-Mills on a Riemann surface of arbitrary genus. We again compute the expectation of a gauge-invariant function $f$ on the space of connections $\mathscr{A}$ using a measure $\mu$ on $\mathscr{A} / \mathscr{G}_{m}$. For a trivial $G=S U(N)$ bundle over a Riemann surface $M$ with base point $m$, the space $\mathscr{A} / G_{m}$ is an affine-linear bundle over a topologically non-trivial base space. If $M$ has genus $g \geqq 1$, the base space is $\operatorname{Path}^{2 g} G$, the set of $2 g$-tuples in $G$ subject to a single relation among the end-point values of the component paths. The measure $\mu$ induces a Gaussian measure on the fibers in $\mathscr{A} / \mathscr{G}_{m}$. Integration over the fibers pushes $\mu$ forward to a measure on $\mathrm{Path}^{2 g} G$, which proves to be the product of Wiener measures on the component paths, subject to the relation on endpoint values. These paths are parametrized by the areas enclosed by a one-parameter family of closed paths on $M$. Conformal transformations of the metric on $M$ act to reparametrize the paths in $\operatorname{Path}^{2 g} G$. We conclude by computing the expectation of a class of Wilson loops. For example, let $g=1$, and let $\mathscr{W}_{1}$ and $\mathscr{W}_{2}$ be Wilson loops given by holonomy about a homotopic pair of simple closed paths in $M$ which intersect only at $m$. For any central function $f$ on $G$, we compute

$$
\left\langle f\left(\mathscr{W}_{i}\right)\right\rangle=\frac{\int f(x) H\left(x y x^{-1} y^{-1} ; 2|M|\right) d x d y}{\int H\left(x y x^{-1} y^{-1} ; 2|M|\right) d x d y},
$$

and

$$
\left\langle f\left(\mathscr{W}_{1}^{-1} \mathscr{W}_{2}\right)\right\rangle=\frac{\int f(\bar{x}) H(\bar{x} ; \Delta t) H\left(\bar{x}^{-1} x y x^{-1} y^{-1} ; 2|M|-\Delta t\right) d \bar{x} d x d y}{\int H\left(x y x^{-1} y^{-1} ; 2|M|\right) d x d y},
$$

where $H$ is the heat kernel on $G,|M|$ is the area of the torus $M, \Delta t$ is twice the area enclosed by the pair of closed paths, and the integrals are with respect to the Haar measure on $G$.

This paper proceeds as follows:

Section 1 defines some notation and conventions.

Section 2 briefly compares the $g \geqq 1$ with the $g=0$ case.

Section 3 realizes $\mathscr{A} / \mathscr{G}_{m}$ as a bundle over Path $^{2 g} G$.

Section 4 describes quantum expectations as integrals over this bundle with respect to the measure $\mu$ and exhibits the Gaussian measure on the fibers of $\mathscr{A} / \mathscr{G}_{m}$.

Section 5 interprets the push-forward of $\mu$ to Path $^{2 g} G$ in terms of Wiener measures on the component paths.

Section 6 discusses the behavior of the measure on $\mathrm{Path}^{2 g} G$ under conformal transformations of the metric on $M$.

Section 7 computes some examples.

\section{Conventions}

On a product bundle, a connection $A$ is a Lie-algebra-valued 1-form, and a gauge transformation $\psi$ is a $G$-valued function. The effect of a gauge transformation on a connection is

$$
\psi \cdot A=\psi^{-1} A \psi+\psi^{-1} \mathbf{d} \psi
$$


The horizontal lift according to a connection $A$ of a curve $\gamma(t)$ in $M$ is a $G$-valued function $U_{A}$ which satisfies the parallel transport equation along $\gamma$

$$
\frac{d}{d t} U_{A}(\gamma)=U_{A}(\gamma) A\left(\frac{d \gamma}{d t}\right)
$$

with $U_{A}(\gamma(0))=1$, the identity element in $G$. Parallel transport about a closed path in $M$ is a Wilson loop. If the closed path begins at $m$, where the gauge transformations are the identity, the corresponding Wilson loops are gauge invariant.

The curvature of a connection is a Lie-algebra-valued 2-form $F_{A}$. The $G$-invariant metric on the Lie algebra combined with a metric $h$ on the base space defines a $G$-invariant metric (,) on Lie-algebra-valued forms. The Yang-Mills action $S(A)$ is a factor of $\frac{1}{4}$ times the integral over the base space of the square in this metric of the curvature.

The metric on $\mathscr{A}$ induces a metric on $\mathscr{A} / \mathscr{G}_{m}$ by distinguishing a natural choice of representative in $T \mathscr{A}$ for each element of $T\left(\mathscr{A} / \mathscr{G}_{m}\right)$. Denoting the tangent to the gauge orbits by $T^{V} \mathscr{A}$, the metric on $A$ defines its orthogonal complement $T^{H} \mathscr{A}$. This distinguishes representatives in $T \mathscr{A}$ for elements of $T\left(\mathscr{A} / \mathscr{G}_{m}\right)$. The induced metric on $\mathscr{A} / \mathscr{G}_{m}$ is the metric on $\mathscr{A}$ applied to these representatives. Explicitly, $T^{V} \mathscr{A}$ is the image of the covariant differential $D_{A}$ on Lie-algebra-valued functions which are 0 at $m$. The orthogonal complement is $\operatorname{ker} D_{A}^{*}$, so the induced metric on $\mathscr{A} / \mathscr{G}_{m}$ is the metric on $\mathscr{A}$ restricted to $\operatorname{ker} D_{A}^{*}$.

We shall represent the surface $M$ as a fundamental domain $D \subset R^{2}$, which will be a regular polygon. Without loss of generality we assume the base point $m$ lies at the center. If $M$ has genus $g$, then the boundary $\partial D$ of $D$ has $4 g$ edges which are identified to each other pairwise with opposite orientations as in Fig. 1.1. The edges correspond to generators of the fundamental group of $M$ (and their inverses) and satisfy the relation

$$
\prod_{i=1}^{2 g} a_{i} b_{i} a_{i}^{-1} b_{i}^{-1}=\mathbf{1}
$$

where successive factors multiply from the right. The usual polar coordinates describe points in the interior of $D$ and extend to $\partial D$ subject to the above identifications.

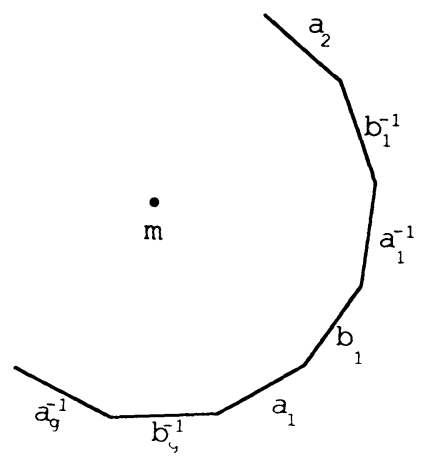

Fig. 1.1. The fundamental domain 
The natural class of connections for physics consists of those whose curvature is bounded. In two dimensions, as Uhlenbeck [6] points out, these connections need not be continuous. The gauge transformations between such connections necessarily are continuous. To make sense of the path integration over the fiber of $\mathscr{A} / \mathscr{G}_{m}$, we must restrict to piecewise $C^{1}$ connections $A$ satisfying the following:

Continuity Condition. On $\partial D$, the component of $A$ in the direction of the tangent to $\partial D$ must respect the identification of the edges. Further, approaching the origin, $A$ must become purely radial. For a connection whose radial part vanishes on $\partial D$, this requires

$$
A_{\theta}(p)=-A_{\theta}\left(p^{-1}\right) \quad \text { and } \quad A_{\theta}(m)=0,
$$

for $p$ any point of $\partial D$.

Note that continuous gauge transformations preserve this condition. Henceforth $\mathscr{A}$ will refer to connections which satisfy the above continuity condition and have finite curvature. Similarly, $\mathscr{G}_{m}$ will refer to gauge transformations between them, which, according to [6], are in $L_{2}^{2}$. Further, Path ${ }^{2 g} G$ will refer to $2 g$-tuples of paths, each component of which lies in $L_{1}^{2}$, so the component paths have finite energy.

\section{Comparing Higher Genus to the Two-Sphere}

The following comparison of the $g \geqq 1$ case with the $g=0$ case may help to orient readers familiar with the treatment of the latter in [2]. For a bundle over a surface of genus $g$, holonomy about a certain family of loops defines a projection from $\mathscr{A} / \mathscr{G}_{m}$ to $\mathrm{Path}^{2 g} G$. With this projection, $\mathscr{A} / \mathscr{G}_{m}$ is a principal bundle over $\mathrm{Path}^{2 g} G$ having an affine linear fiber. Along each fiber, the Yang-Mills action is quadratic. In the $g=0$ case, $\mathscr{A} / \mathscr{G}_{m}$ was similarly a principal bundle with affine linear fiber and base space $\Omega G$, based loops in $G$. Note that a based loop is a path subject to two conditions: each endpoint must take the value 1 .

The integration over the affine linear fiber, and the interpretation of the result as a path integral over $\mathrm{Path}^{2 g} G$ closely follow the $g=0$ case. The need to consider arbitrary metrics introduces some complications in the interpretation of the measure on Path $^{2 g}$ G. Working these out leads to a characterization of the conformal properties of the theory which also applies to the $g=0$ case.

\section{The Projection from $\mathscr{A} / \mathscr{G}_{m}$ to Path $^{2 g} G$}

Consider a path in $D$ which proceeds radially from the origin $m$ to a point $p$ on $a_{1}$ and back radially to $m$ from the corresponding point $p^{-1}$ on $a_{1}^{-1}$. Figure 3.1 illustrates one such path. Relative to a point of the fiber over $m$, fixed once for all connections and all paths through $m$, the holonomy of a given connection about the above path corresponds to an element of $G$. As the point $p$ varies along $a_{1}$, the corresponding holonomies describe a path in $G$. Denote this path by $\alpha_{1}$. Note that although the two vertices of $a_{1}$ correspond to the same point of $M$, the endpoint values of $\alpha_{1}$ will in general be distinct elements of $G$. Radial paths to points in 


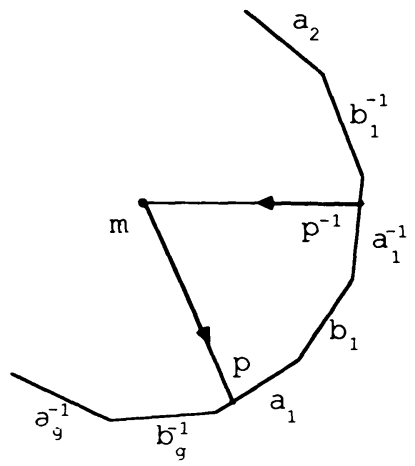

Fig. 3.1. A typical radial closed path

the edges $b_{1}, a_{2}, b_{2}, \ldots, a_{g}, b_{g}$ and from their inverses similarly define paths in $G$ and thus a $2 g$-tuple of paths $\left(\alpha_{1}, \beta_{1}, \ldots, \alpha_{g}, \beta_{g}\right)$. We shall use $c_{i}$ to refer to a generic edge in $\left\{a_{1}, b_{1}, \ldots, a_{g}, b_{g}\right\}$. Its inverse will be $c_{i}^{-1}$, and $\gamma_{i}$ will denote the corresponding path in $G$. Similarly, $\vec{\gamma}$ will denote the $2 g$-tuple of paths. Note that henceforth $\gamma_{i}$ refers to a path in $G$, not in $M$.

The map which takes a connection $A$ to $\vec{\gamma} \in \mathrm{Path}^{2 g} G$ is invariant under the action of $\mathscr{G}_{m}$, since these gauge transformations are the identity at the origin $m$ of $D$. Thus this map defines a map $\xi: \mathscr{A} / \mathscr{G}_{m} \rightarrow$ Path $^{2 g} G$. Let $\gamma_{i}(0)$ and $\gamma_{i}(1)$ denote the values of $\gamma_{i}$ obtained by choosing the point $p$ to be, respectively, the first and the second endpoint of $c_{i}$ in the usual counter-clockwise orientation of $\partial D$.

Theorem 3.1. $\mathscr{A} / \mathscr{G}_{m}$ is a principal bundle with projection $\xi$ and affine linear fiber. The base space is $\mathrm{Path}^{2 g} G$, the set of $2 g$-tuples of paths $\vec{\gamma}$ in $G$ satisfying the relation

$$
\prod_{i=1}^{g} \alpha_{i}(0) \beta_{i}(1)^{-1} \alpha_{i}(1)^{-1} \beta_{i}(0)=1
$$

where successive factors multiply from the right.

The need for the relation stems from the fact that while the family of closed paths whose holonomies define the projection $\xi$ covers $D$, some points lie on more than one such path. These points constitute the radial paths between the origin and the $4 g$ vertices. The relation holds true because the left-hand side is holonomy about a particular concatenation of such paths which reduces to the identity path. To prove the relation, first show directly that the general factor

$$
\alpha_{i}(0) \beta_{i}(1)^{-1} \alpha_{i}(1)^{-1} \beta_{i}(0)
$$

is the holonomy about a radial loop out to the first vertex of $a_{i}$ and back from the second vertex of $b_{i}^{-1}$. The relation then follows by induction.

To see that $\xi$ is onto, consider any element $\vec{\gamma}$ of $\operatorname{Path}^{2 g} G$. To construct a representative $A$ of a class $[A]$ which $\xi$ maps to this $2 g$-tuple, begin by prescribing the parallel transports by $A$ along radial paths from $m$ to the boundary. For $p \in c_{i}$, let $U(p)$ denote an element of $G$ which will be parallel transport along the radial path from $m$ to $p$. Then,

$$
\gamma_{i}(p)=U(p) U\left(p^{-1}\right)^{-1}
$$

where $p$ parametrizes $\gamma_{i}$ in an obvious fashion. 
Suppose the outward parallel transport to the second end of $b_{i}^{-1}$ has been assigned the value $U_{i}$. Then, parallel transport out the first end of $b_{i}$ must be $\beta_{i}(0) U_{i}$ to ensure the product of the first parallel transport with the inverse of the second yields $\beta_{i}(0)$. Now, the first end of $b_{i}$ is also the second end of $a_{i}$, so to get $\alpha_{i}(1)$ from parallel transports, the parallel transport out to the first end of $a_{i}^{-1}$ must be $\alpha_{i}(1)^{-1} \beta_{i}(0) U_{i}$. Continuing in this tortuous fashion ultimately requires parallel transport out to the first end of $a_{i}$ to be $\alpha_{i}(0) \beta_{i}(1)^{-1} \alpha_{i}(1)^{-1} \beta_{i}(0) U_{i}$. Note that the first end of $a_{i}$ is also the second end of $b_{i-1}$, so this determines $U_{i-1}$. By induction, this implies a consistency requirement,

$$
\left(\prod_{i=1}^{g} \alpha_{i}(0) \beta_{i}(1)^{-1} \alpha_{i}(1)^{-1} \beta_{i}(0)\right) U_{g}=U_{g}
$$

which is exactly the relation restricting elements of Path $^{2 g} G$. Thus, a choice of $U_{g} \in G$ determines $4 g$ group elements which will be the parallel transports by $A$ outward to the vertices of $\partial D$.

For $p \in c_{i}$, choose $U(p)$ so that $U$ is a piecewise continuous path in $G$ connecting the two endpoint values. Then Eq. (3.1) determines $U\left(p^{-1}\right)$. This completes the specification of the radial parallel transports of the connection $A$.

To construct a connection having these parallel transports, let

$$
A_{r}(r, \theta)=\zeta(r) \ln U(p(\theta)),
$$

where $p(\theta)$ is the point of $c_{i}$ determined by $\theta$, and $\zeta$ is a smooth function which is zero outside of a sufficiently small interval and has integral 1 . The outward parallel transport to a radius $r$ by any connection having this radial part is

$$
\exp \left[\int_{0}^{r} \zeta(\bar{r}) d \bar{r} \ln U\right] .
$$

In particular, the outward parallel transports to the boundary are the above function $U$.

Finally, define $A=A_{r} \mathrm{~d} r$ to obtain a connection satisfying the continuity condition and representing a class in $\mathscr{A} / \mathscr{G}_{m}$ which projects under $\xi$ to $\vec{\gamma}$. This completes the proof that $\xi$ maps onto $\mathrm{Path}^{2 g} G$.

Given any point $[A]$ in the fiber over $\vec{\gamma}$, the fiber consists of all points of the form $[A+\tau]$, where $\tau \in \wedge^{1}(M, \mathbf{g})$ has radial component equal to zero. (The symbol g denotes the Lie algebra of $G$.) Clearly, every point of this form lies in the fiber. To prove that every point of the fiber has this form, let $[B]$ be another point of the fiber. Let $A$ and $B$ be connections representing these classes. The claim is that there is a gauge transformation $\psi$ and a one-form $\tau$ with vanishing radial component such that $B=\psi \cdot(A+\tau)$.

Consider again the family of closed paths in $M$ whose holonomies define $\xi$. The gauge transformation $\psi$ takes lifts of these paths by $A$ to lifts by $B$. Consider the radial path out to $p \in c_{i}$ and back from $p^{-1}$. It lifts by $A$ and $B$ differ by an automorphism of the fibers over the path. Since $A$ and $B$ agree on holonomy, this automorphism is the identity over $m$. Forming the analogous automorphism of the fibers over each such closed path yields an automorphism, which is the identity over $m$, of the bundle over $M$. Since the radial paths to the vertices occur more than once in the family of closed paths, this automorphism might not be continuous. 
However, explicitly checking the different definitions of the automorphism along such paths shows they agree. This automorphism is thus well-defined and is the desired gauge transformation $\psi$. Since $\psi$ takes radial lifts by $A$ to radial lifts by $B$, $A$ and $\psi^{-1} \cdot B$ must differ by a one-form $\tau$ which is zero in the radial direction. If $A$ and $B$ satisfy the continuity condition, then so does $\psi^{-1} \cdot B$ and, therefore, $\tau$. Thus the claim is valid.

Defining, on $\wedge^{1}$, local projections $P_{r}$ and $P_{\theta}$ to components, the fiber is isomorphic to $\operatorname{ker} P_{r}$. The choice of the class $[A]$ serves as a choice of origin in the affine fiber, making explicit the isomorphism. The next section uses this explicit isomorphism, with a special choice of [A], to integrate over the fiber.

\section{The Integration Over the Fibers in $\mathscr{A} / \mathscr{G}_{m}$}

The measure $\mu$ on $\mathscr{A} / \mathscr{G}_{m}$ to use in computing quantum expectations is, up to a constant, the push-forward of the measure on $\mathscr{A}$ which the metric induces. We describe the measure $\mu$ in a general context in [2, Sect. 1], where we also discuss its equivalence with the measure given by the Faddeev-Popov gauge-fixing prescription. With $\rho: \mathscr{A} \rightarrow \mathscr{A} / \mathscr{G}_{m}$ denoting the usual projection, Theorem 3.1 of [2] carries over to give the restriction of $\mu$ to the fiber. In polar coordinates on $D$ this is

\section{Theorem 4.1.}

$$
\int_{\xi^{-1}(\vec{\gamma})} f([A]) \mu_{\text {fiber }}=\operatorname{det}^{1 / 2}\left(D_{A}^{*} P_{r} D_{A}\right) \int_{\operatorname{ker} P_{r}} f(A+\tau) e^{-S(A+\tau)} \mathscr{D} \tau,
$$

where $A$ is an arbitrary connection representing a point in the fiber $\xi^{-1}(\vec{\gamma})$.

As in the genus- 0 case, we would like to select an origin in the fiber by finding a connection $\tilde{A}$ for which the action $S(\tilde{A}+\tilde{\tau})$ is purely quadratic in $\tilde{\tau} \in \operatorname{ker} P_{r}$. This requires $\left\langle F_{\tilde{A}}, D_{\tilde{A}} \tau\right\rangle=0$ for all $\tau \in \operatorname{ker} P_{r}$.

Theorem 4.2. The condition $\left\langle F_{\tilde{A}}, D_{\tilde{A}} \tau\right\rangle=0$ for all $\tau \in \operatorname{ker} P_{r}$ determines a unique section of $\mathscr{A} / \mathscr{G}_{m}$ over $\mathrm{Path}^{2 g} G$.

To prove this, integrate the condition by parts, keeping track of the boundary term and the continuity condition on $\tau$, to obtain the pair of conditions

$$
P_{\theta} * D_{\tilde{A}} * F_{\tilde{A}}=0
$$

and, for $p \in \partial D$,

$$
* F_{\tilde{A}}\left(p^{-1}\right)=* F_{\tilde{A}}(p) .
$$

As in the genus-0 case, there is a 1 -form $\tau^{\prime} \in \operatorname{ker} P_{r}$ satisfying $P_{\theta} * D_{A} * D_{A} P_{\theta} \tau^{\prime}=$ $P_{\theta} * D_{A} * F_{A}$, and shifting any connection $A$ by $\tau^{\prime}$ yields a connection $\tilde{A}_{0}=A-\tau^{\prime}$ satisfying the first of these conditions. However, for $g \geqq 1, \operatorname{ker} P_{\theta} * D_{A} * D_{A} P_{\theta}$ is not trivial, so $\tau^{\prime}$ is not unique. We will exploit this indeterminancy to satisfy the second of the pair of conditions. Choose any $\tilde{A}_{0}$ as above, and consider, as candidates for the connection $\widetilde{A}$, connections of the form $\widetilde{A}_{0}-\tau_{l}$, with $\tau_{l} \in \operatorname{ker} P_{\theta} * D_{A} * D_{A} P_{\theta}$. Since $* F_{\tilde{A}}=* F_{\tilde{A}_{0}}-* D_{A} \tau_{l}$, the condition on $F_{\tilde{A}}$ restricts $D_{A} \tau_{l}$. The 1 -form $\tau_{l}$ is covariantly linear along radii and vanishes in the radial direction. Since $\tau_{l}$ must 
vanish at $m$, its differential $D_{A} \tau_{l}$, which is covariantly constant along radii, completely determines $\tau_{l}$. Further, the continuity condition on $\tau_{l}$ restricts $D_{A} \tau_{l}$ on the boundary to satisfy

where

$$
v\left(p^{-1}\right) * D_{A} \tau_{l}\left(p^{-1}\right)=-v(p) * D_{A} \tau_{l}(p)
$$

$$
v(p)=\int_{\substack{\text { radius } \\ \text { through } p}} \sqrt{h} d r .
$$

The length of the radial path enters, because $\tau_{l}(p)$ is the integral over the lift of this path of the constant $D_{A} \tau_{l}$. The condition on $F_{\tilde{A}}$ and Eq. (4.1) completely determine $D_{A} \tau_{l}$ to be

$$
* D_{A} \tau_{l}(p)=\left[1+\frac{v(p)}{v\left(p^{-1}\right)}\right]^{-1}\left[* F_{\tilde{A}_{0}}(p)-* F_{\tilde{A}_{0}}\left(p^{-1}\right)\right]
$$

This, in turn, determines $\tau_{l}$ and hence $\tilde{A}$. Gauge transformations affect each side of this equation by pointwise conjugation, and respect the identification of $p$ with $p^{-1}$, so the class $[\tilde{A}]$ does not depend, in this respect, on the choice of representative $\tilde{A}_{0}$ for $\left[\tilde{A}_{0}\right]$. Further, straight-forward checking verifies that any choice of the connection $\tilde{A}_{0}$ satisfying the first of the pair of conditions leads to the same class $[\tilde{A}]$ for the origin in the fiber. Taken together these classes define the global section over Path ${ }^{2 g} G$.

With $\tilde{A}$ serving as a choice of origin, Theorem 4.1 becomes

$$
\int_{\xi^{-1}(\tilde{\gamma})} f([\tilde{A}]) \mu_{\text {fiber }}=\operatorname{det}^{1 / 2}\left(D_{A}^{*} P_{r} D_{A}\right) \int f(\tilde{A}+\tilde{\tau}) e^{-(1 / 4)\|F \tilde{A}\|^{2}} e^{-(1 / 4)\left\|D_{A} \tilde{\tau}\right\|^{2}} D \tilde{\tau} .
$$

Now 'change variables from $\tilde{\tau}$ to $D_{A} \tilde{\tau}$. This introduces a Jacobian factor of $\left(\operatorname{det}^{\prime}\right)^{-1 / 2}\left(P_{\theta} D_{A}^{*} D_{A} P_{\theta}\right)$. If $f=1$, this integral gives the factor relating the push-forward measure $\xi_{*} \mu$ to $\operatorname{vol}_{\mathrm{Path}^{2 g} G}$, the measure on paths which the metric on $\mathscr{A}$ induces. The push-forward is

$$
\xi_{*} \mu=\left(\int \mu_{\text {fiber }}\right) \operatorname{vol}_{\mathrm{Path}^{2 g} \mathrm{G}} \text {. }
$$

The next section will describe the volume element $\operatorname{vol}_{\mathrm{Path}^{2 g_{G}}}$ explicitly. With

we have shown

$$
\hat{S}(\tilde{A})=\frac{1}{4}\left\|F_{\tilde{A}}\right\|^{2},
$$

Corollary 4.2.1.

$$
\xi_{*} \mu=e^{-\hat{s}} \frac{\operatorname{det}^{1 / 2}\left(D_{A}^{*} P_{r} D_{A}\right)}{\left(\operatorname{det}^{\prime}\right)^{1 / 2}\left(P_{\theta} D_{A}^{*} D_{A} P_{\theta}\right)} \operatorname{vol}_{\operatorname{Path}^{2 q} G}
$$

More generally, if $f$ depends polynomially on $D_{A} \tilde{\tau}$, the Gaussian integrals yield the same determinant factors times a function $\hat{f}(\hat{A})$. Thus, for the integral over the fiber,

\section{Corollary 4.2.2.}

$$
\int f \mu_{\mathrm{fiber}}=\hat{f} e^{-\hat{s}} \frac{\operatorname{det}^{1 / 2}\left(D_{A}^{*} P_{r} D_{A}\right)}{\left(\operatorname{det}^{\prime}\right)^{1 / 2}\left(P_{\theta} D_{A}^{*} D_{A} P_{0}\right)}
$$


Since the section represented by $\tilde{A}$ is unique, $\hat{f}$ and $\hat{S}$ must be functions only of the base point $\vec{\gamma}$. The next section contains an explicit computation of $\hat{S}$, showing that it is the energy action on $\mathrm{Path}^{2 g} G$.

\section{The Integration Over Path ${ }^{2 g} G$}

This section re-describes the results of integration over the fiber as $\hat{f}$ times an explicit measure on $\mathrm{Path}^{2 g} G$. Evaluating $\hat{S}$, the ratio of determinants, and $\operatorname{vol}_{\mathrm{Path}^{2 g} \mathrm{G}}$ will yield

\section{Theorem 5.1.}

$$
\int_{\xi^{-1}(\vec{\gamma})} f([A]) \mu_{\mathrm{fiber}}=\hat{f} \exp \left[-\frac{1}{2} \sum_{i=1}^{2 g} \int\left|\frac{d \gamma_{i}}{d t_{i}}\right|^{2}\right],
$$

up to a determinant factor depending only on the metric h. Here $\hat{f}$ is defined as in Corollary 4.2.2. The parameter $t_{i}$, replacing $p$, is twice the area on $M$ between the closed paths whose holonomies gave $\gamma(0)$ and $\gamma(p)$, oriented so the path through $p$ is reversed. Further, up to a similar determinant factor,

$$
\xi_{*} \mu=\exp \left[-\frac{1}{2} \sum_{i=1}^{2 g} \int\left|\frac{d \gamma_{i}}{d t_{i}}\right|^{2}\right] \mathscr{D} \vec{\gamma}
$$

with the endpoint relation of Theorem 3.1 restricting $\vec{\gamma}$.

For quantum expectations, which are a normalization constant times the integral with respect to $\mu$, this gives

\section{Corollary 5.1.1.}

$$
\langle f\rangle=\frac{1}{Z_{0}} \int_{\text {Path }^{2} \theta_{G}} \hat{f}(\vec{\gamma}) \exp \left[-\frac{1}{2} \sum_{i=1}^{2 g} \int\left|\frac{d \gamma_{i}}{d \tau_{i}}\right|^{2}\right] \mathscr{D} \vec{\gamma} .
$$

Remark 5.1. The parameters $t_{i}$ could each change by a constant without affecting the above result. We have chosen this constant so that the smallest value of $t_{i}$ is zero. This corresponds to measuring areas relative to the closed path determined by radii through the first endpoint of $c_{i}$ and the second endpoint of $c_{i}^{-1}$. See

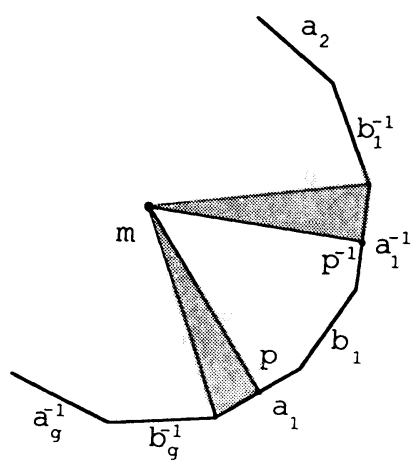

Fig. 5.1. The area $\frac{1}{2} t_{i}(p)$ 
Fig. 5.1. Measuring area relative to another path homotopic to this one would change $t_{i}$ by a constant area.

5.1 Evaluating $\hat{S}$. The main point is to show $\hat{S}$ reduces to the energy action on paths. This is a consequence of Stoke's Law, which connects $F_{\tilde{A}}$, and hence $\hat{S}$, to the energy action on $\mathrm{Path}^{2 g} G$ as follows:

Corollary to Stoke's Law. The action which the Yang-Mills action on $\mathscr{A}$ induces on the base space $\mathrm{Path}^{2 g} G$ of $\mathscr{A} / \mathscr{G}$ is the energy action

$$
\hat{S}=\frac{1}{2} \sum_{i=1}^{2 g} \int_{0}^{T_{i}}\left|\frac{d \gamma_{i}}{d t_{i}}\right|^{2} d t_{i},
$$

where $T_{i}$ is twice the area closed by radii through the endpoints of $c_{i}$ and $c_{i}^{-1}$.

Consider the radial closed paths, $\sigma_{p}$ and $\sigma_{(p+d p)}$, whose holonomies are $\gamma_{i}(p)$ and $\gamma_{i}(p+d p)$, respectively. With the appropriate orientations, their product is a closed path $\sigma_{(p+d p)} \sigma_{p}^{-1}$ whose holonomy is $\gamma_{i}(p+d p) \gamma_{i}(p)^{-1}$. Stoke's Law relates this to the integral of the curvature over the region between the lifts of the closed paths. At first order in $d p$, the relation is

$$
\dot{\gamma}_{i} \gamma_{i}^{-1}=-\int_{\sigma_{p}} * F_{A} \sqrt{h} d r
$$

where the curvature is evaluated at the lift by $A$ of the closed path in $M$. For the connection $\tilde{A}$, whose curvature is covariant constant, the right-hand side becomes

$$
\begin{aligned}
\int_{\sigma_{p}} * F_{\tilde{A}} \sqrt{h} d r & =\int_{\substack{\text { radius } \\
\text { through } p}} * F_{\tilde{A}} \sqrt{h} d r+\int_{\substack{\text { radius } \\
\text { through } p^{-1}}} * F_{\tilde{A}} \sqrt{h} d r \\
& =* F_{\tilde{\boldsymbol{A}}}(p) v(p)+* F_{\tilde{\boldsymbol{A}}}\left(p^{-1}\right) v\left(p^{-1}\right) \\
& =* F_{\tilde{\boldsymbol{A}}}(p)\left[v(p)+v\left(p^{-1}\right)\right] .
\end{aligned}
$$

Note that the sign on the second term is correct. The minus sign from the orientation of the radius cancels one from the direction of the infinitesimal displacement $d p$. Thus, $* F_{\tilde{A}}(p)=-\left[v(p)+v\left(p^{-1}\right)\right]^{-1} \dot{\gamma}_{i} \gamma_{i}^{-1}$ for $p \in \partial D$. Substituting into the definition of $\hat{S}$, Eq. (4.2), and performing the radial integral gives the expression

$$
\hat{S}=\frac{1}{4} \sum_{i=1}^{2 g} \int_{c_{i}}\left|\dot{\gamma}_{i}\right|^{2}\left[v(p)+v\left(p^{-1}\right)\right]^{-1} d p
$$

Now change the integration variable from $p$ to

$$
t_{i}=2 \int_{0}^{p}\left[v(\bar{p})+v\left(\bar{p}^{-1}\right)\right] d \bar{p}
$$

recalling that

$$
\dot{\gamma}_{i}=\frac{d \gamma}{d p}
$$

This yields the corollary. 
5.2. Determinants. The proof of Eq. (4.2) of [2] carries over, with obvious modifications, to prove the analogous result for the ratio of determinants in Corollaries 4.2.1 and 4.2.2. That is,

$$
\frac{\operatorname{det}^{1 / 2}\left(D_{A}^{*} P_{r} D_{A}\right)}{\left(\operatorname{det}^{\prime}\right)^{1 / 2}\left(P_{\theta} D_{A}^{*} D_{A} P_{\theta}\right)}=1 .
$$

The continuity condition is crucial to comparing the ranges, and hence the spectra of the operators in question.

5.3. The Volume Element on $\mathrm{Path}^{2 g} G$. The measure $\mu$ is the product of the measure $\mu_{\text {fiber }}$ and the measure $\operatorname{vol}_{\text {Path }^{2 g} G}$, where the metric in $\mathscr{\sim}$ on $T_{\tilde{A}}^{H}\left(\mathscr{A} / \mathscr{G}_{m}\right)$, the orthogonal complement on the fiber directions at $\tilde{A}$, defines the metric inducing vol $_{\text {Path }^{2 g} G}$. The map $\xi$ acts as a change of variables from $\mathscr{A} / \mathscr{G}_{m}$ to $\mathrm{Path}^{2 g} G$. On the tangent spaces, the change of variables is

$$
\left.\mathbf{d} \xi\right|_{H}: T^{H}\left(\mathscr{A} / \mathscr{G}_{m}\right) \rightarrow T\left(\mathrm{Path}^{2 g} G\right) \sim \mathrm{Path}^{2 g} \mathbf{g} .
$$

Thus, vol $_{\text {Path }^{2 g_{G}}}$ is $\mathscr{D} \vec{\gamma}$ times the Jacobian $\operatorname{det}^{-1 / 2}\left(\left.\left.\mathbf{d} \xi\right|_{H} ^{*} \mathbf{d} \xi\right|_{H}\right)$ of this mapping.

We will show the Jacobian is constant on $\operatorname{Path}^{2 g} G$. First, we compute $\left.\mathbf{d} \xi\right|_{H}$ on $\eta \in T \mathscr{A}$ representing an element of $T^{H}\left(\mathscr{A} / \mathscr{G}_{m}\right)$. Since $\eta$ must be orthogonal to the fiber directions,

$$
\eta=P_{r} \eta, \quad \text { and }\left\langle P_{r} D_{A} f, \eta\right\rangle=0
$$

for all continuous functions $f$ vanishing at the origin. Upon integration by parts, the condition on $\eta$ becomes,

$$
-\int_{D} f \wedge D_{A} P_{\theta} * \eta+\int_{\partial D} f \wedge P_{\theta} * \eta=0
$$

For this to hold for all allowed $f, \eta$ must satisfy

$$
D_{A} P_{\theta} * \eta=0 \text { and } P_{\theta} * \eta\left(p^{-1}\right)=-P_{\theta} * \eta(p),
$$

since continuity requires that $f\left(p^{-1}\right)=f(p)$ for $p \in \partial D$. Thus $* \eta$ is covariant constant along radii, and, on $\partial D$,

$$
\eta_{r}\left(p^{-1}\right)=-\eta_{r}(p)
$$

Explicitly,

$$
\eta_{r}(r, p)=C^{\prime}(r, p) U^{-1}(r, p) \hat{\eta}(p) U(r, p),
$$

where $U(r, p)$ is parallel transport along the radius through $p, \hat{\eta}$ may be any map from $\partial D$ to $\mathbf{g}$ for which the above condition holds, and

$$
C^{\prime}\left(h^{r r} \sqrt{h}\right)^{-1} \text {. }
$$

Note that the values of $\hat{\eta}$ on the edges $c_{i}$ determine its values on the edges $c_{i}^{-1}$. In fact,

$$
\hat{\eta}\left(p^{-1}\right)=-\frac{C^{\prime}(p)}{C^{\prime}\left(p^{-1}\right)} \gamma_{i}(p)^{-1} \hat{\eta}(p) \gamma_{i}(p) .
$$

The map $\left.\mathbf{d} \xi\right|_{H}$ from the tangent $\eta$ to a tangent to Path $^{2 g} G$ at $\vec{\gamma}$ follows from 
the effect of $\eta$ on parallel transport:

$$
\begin{aligned}
\left.\mathbf{d} \xi\right|_{H}(\eta) & =\left.\frac{d}{d t}\right|_{t=0}(\xi \circ \rho)(A+t \eta) \\
& =\left.\frac{d}{d t}\right|_{t=0} U_{A+t \eta}(p)\left[U_{A+t \eta}\left(p^{-1}\right)\right]^{-1}
\end{aligned}
$$

To first order in $t$,

$$
U_{A+t \eta} \sim U_{A}\left[1+t U_{A}^{-1}\left(\int_{0}^{r} U_{A} \eta_{r} U_{A}^{-1} d \bar{r}\right) U_{A}\right],
$$

as follows directly from the parallel transport equation (1.1). For $\eta$ satisfying (5.1), this means

$$
\xi(A+t \eta) \sim \xi(A)\left\{\mathbf{1}-t\left[C(p)+C\left(p^{-1}\right)\right] \hat{\eta}\left(p^{-1}\right)\right\}
$$

where

$$
C(p)=\int_{\substack{\text { radius } \\ \text { through } p}} C^{\prime}(r, p) d r .
$$

Thus, the change of variables is,

$$
\left.\mathbf{d} \xi\right|_{\boldsymbol{H}}(\eta)=-\left[C(p)+C\left(p^{-1}\right)\right] \hat{\eta}\left(p^{-1}\right)
$$

We use this to show

Theorem 5.2. The determinant of $\left.\left.\mathbf{d} \xi\right|_{H} ^{*} \mathbf{d} \xi\right|_{H}$ is the determinant of multiplication by

$$
\frac{(C+\bar{C})^{2}}{C+\bar{C}\left(\frac{C^{\prime}}{\overline{C^{\prime}}}\right)}
$$

on functions from $\left\{c_{i}\right\}$ to $\mathbf{g}$, where $\bar{C}(p)=C\left(p^{-1}\right)$.

To prove the theorem, consider the set of functions $\{\hat{\eta} \tilde{C}\}$, where the $\hat{\eta}$ are an orthonormal basis of $\mathrm{Path}^{2 g} \mathrm{~g}$ and

$$
\tilde{C}(p)=\left\{C(p)+C\left(p^{-1}\right)\left[\frac{C^{\prime}(p)}{C^{\prime}\left(p^{-1}\right)}\right]^{2}\right\}^{-1 / 2} .
$$

As it stands, these functions are only defined for arguments $p \in c_{i}$. Extend them to functions on all of $\partial D$ by requiring $\hat{\eta} \widetilde{C}$ to satisfy Eq. (5.3). With the above definition of $\widetilde{C}$ these extended functions form a basis $\left\{\eta^{i}\right\}$ for $T^{H}\left(\mathscr{A} / \mathscr{G}_{m}\right)$, since

$$
\begin{aligned}
\left\langle\eta^{i}, \eta^{j}\right\rangle & =\iint\left(C^{\prime} U^{-1} \hat{\eta}^{i} \tilde{C} U, U^{-1} \hat{\eta}^{j} \tilde{C} U\right) h^{r r} \sqrt{h} d r d \theta \\
& =\int C(\theta)\left(\hat{\eta}^{i} \tilde{C}, \hat{\eta}^{j} \tilde{C}\right) d \theta \\
& =\sum_{i} \int_{c_{i}}\left\{C(p)+C\left(p^{-1}\right)\left[\frac{C^{\prime}(p)}{C^{\prime}\left(p^{-1}\right)}\right]^{2}\right\}[\tilde{C}(p)]^{2}\left(\hat{\eta}^{i}, \hat{\eta}^{j}\right) d p \\
& =\delta^{i j} .
\end{aligned}
$$


In this orthonormal basis, the $i j^{\text {th }}$ element of the matrix for $\left.\left.\mathbf{d} \xi\right|_{H} ^{*} \mathbf{d} \xi\right|_{H}$ is, by Eq. (5.4),

$$
\left\langle\left.\mathbf{d} \xi\right|_{H}\left(\eta^{i}\right),\left.\mathbf{d} \xi\right|_{H}\left(\eta^{j}\right)\right\rangle=\sum_{i} \int\left(\hat{\eta}^{i},\left[C(p)+C\left(p^{-1}\right)\right]^{2}[\tilde{C}(p)]^{2} \hat{\eta}^{j}\right) d p
$$

Theorem 5.2 follows immediately.

This multiplication operator does not depend on $\vec{\gamma}$, so the determinant is constant on $\mathrm{Path}^{2 g} G$, as claimed. Hence, up to this constant, integration with respect to vol $_{\text {Path }^{2 g} G}$ is integration with respect to $\mathscr{D} \vec{\gamma}$.

Replacing each term in Corollaries 4.2.1 and 4.2.2 with its corresponding expression in terms of $\vec{\gamma}$ completes the proof of Theorem 5.1. Corollary 5.1.1 follows from the observation that the integral with respect to $\mu$ is the integral over each fiber with respect to $\mu_{\text {fiber }}$ followed by integration over $\mathrm{Path}^{2 g} G$ with respect to vol $_{\mathrm{Path}^{2} g_{G}}$.

Corollary 5.1.1 completely specifies the expectation of the function $f$ in terms of an expectation $\hat{f}$ in the Gaussian measure on the fiber over each point of Path $^{2 g} G$ and a conditional Wiener measure on functions of Path ${ }^{2 g} G$. In Sect. 7, we explictly compute an example to indicate how to interpret the path integration over Path ${ }^{2 g} G$ in terms of heat kernels on $G$. As a preliminary, we describe the Wiener measure which respects the relation on the endpoints in $\mathrm{Path}^{2 g} G$.

If the endpoint values of each component path $\gamma_{i}$ were fixed, the right-hand side of Corollary 5.1.1 would be the product of conditional Wiener measures on each component. To obtain a measure on $\operatorname{Path}^{2 g} G$, consider the map $\sigma:$ Path $^{2 g} G \rightarrow G^{4 g}$ which takes each component path to its endpoint values. The product of conditional Wiener measures on each path defines a measure on $\sigma^{-1}(x)$ for each point $x \in G^{4 g}$. Let $\lambda: G^{4 g} \rightarrow G$ be given by the left-hand side of the relation in Theorem 3.1, so that Path ${ }^{2 g} G$ is $\sigma^{-1}\left(\lambda^{-1}(1)\right)$. The product of Haar measures defines a natural measure on $\lambda^{-1}(\mathbf{1})$. Combining the conditional Wiener measure on $\sigma^{-1}(x)$ for each $x \in \lambda^{-1}(1)$ with the Haar measure on $\lambda^{-1}(1)$ yields a measure on $\mathrm{Path}^{2 g} G$. We interpret the path integral on the right-hand side of Corollary 5.1.1 to be integration using this measure.

5.4. The Genus-0 Case. In [2], we fixed the metric to be the standard metric on $S^{2}$. Relaxing this restriction, and repeating that analysis leads to

$$
\xi_{*} \mu_{S_{2}}=\exp \left[-\frac{1}{2} \int_{0}^{2\left|S^{2}\right|}\left|\frac{d \gamma}{d t}\right|^{2}\right] \mathscr{D} \gamma
$$

where $\gamma \in \Omega G$ and the parameter $t$ is twice the area bounded by the path whose holonomy gave $\gamma(t)$. The modification to the expression for the integral over the fibers is similar.

In [5], Sengupta derives, for the $g=0$ cases, expectations of functions of Wilson loops, starting from a stochastic parallel transport equation. He shows these can be computed using conditional Wiener measure on paths in $G$ parametrized by areas of a 1-parameter family of closed paths on $S^{2}$. The family of closed paths is a particular homotopy from the constant path at the north pole to the closed path whose homotopy is the Wilson loop in question. Except for the factor 2 multiplying $\left|S^{2}\right|$, the above equation agrees with his result as it applies to the case of the closed paths bounded by longitudes. We leave for the future the task of 
extracting the more general result from Theorem 4.1 of [2], although the above equation tells most of the story. In the higher-genus case, Theorem 5.1 shows a modification of this statement applies to functions of the Wilson loops $\left\{\gamma_{i}\left(t_{i}\right)\right\}$. The homotopy is from a representative of a generator of $\pi_{1}(M)$ to the path in $M$ whose holonomy is $\gamma_{i}\left(t_{i}\right)$.

\section{Conformal Properties}

Conformal transformations of the metric on $M$ affect the measure $\xi_{*} \mu$ onPath $^{2 g} G$ through the parameter $t_{i}$. They also affect the overall normalization constant. We first describe the former effect.

Theorem 6.1. Under a conformal transformation, the paths in $\mathrm{Path}^{2 g} \mathrm{G}$ are reparametrized. The measure $\xi_{*} \mu$, which is the product of Wiener measures on each path in $\mathrm{Path}^{2 g} G$, is taken to the product of Wiener measures, in the new parametrization, on each path.

The parameter $t_{i}$ of Theorem 5.1 is twice the area bounded by an oriented, closed path on $M$. Under a conformal transformation this area will change. If the transformation takes $h_{\mu \nu}$ to $e^{\phi} h_{\mu \nu}$, then it takes the parameter $t_{i}$ to the new parameter $t_{i}^{\phi}$, related by

$$
\frac{d t_{i}^{\phi}}{d t_{i}}=\frac{\int e^{\phi} \sqrt{h}}{\int \sqrt{h}},
$$

and $t_{i}^{\phi}=0$ when $t_{i}=0$. The integrals are over the radial closed path in $M$ whose holonomy gave $\gamma_{i}\left(t_{i}\right)=\gamma_{i}\left(t_{t}^{\phi}\right)$. The effect of a global change of scale is to speed up (or slow down) the rate at which each of the $2 g$ component paths are traced out. In general, a conformal transformation will change this rate at each point of each path independently. That the transformation takes $\xi_{*} \mu$ to the product of Wiener measures on the paths with the new parametrization follows immediately from Theorem 5.1.

The normalization constant contains two metric-dependent factors. The determinant of $\left.\left.\mathbf{d} \xi\right|_{H} ^{*} \mathbf{d} \xi\right|_{H}$ is invariant under conformal transformations, since the function $C^{\prime}$ of Eq. (5.2) is invariant. The other factor is a normalization constant in the path-integral expression for the Wiener measure. In the following section we will explicitly compute this constant, which turns out to be

$$
\int H\left(x_{1} y_{1} x_{1}^{-1} y_{1}^{-1} \cdots x_{g} y_{g} x_{g}^{-1} y_{g}^{-1} ; 2|M|\right) d x_{1} d y_{1} \cdots d x_{g} d y_{g}
$$

where $H$ is the heat kernel on $G,|M|$ denotes the area of $M$, and the integrals are over $G$.

\section{Some Examples}

For a simple example illustrating the integration of the path integral over $\operatorname{Path}^{2 g} G$ as Wiener measure, take $M$ to be the torus of genus 1 . For a given connection $A$, consider the Wilson loops which are $\alpha(p)$, and $\beta(q)$, where $p \in a, q \in b$, and 
$(\alpha, \beta) \in \operatorname{Path}^{2 g} G$ is the image of $A$ under $\xi \circ \rho$. We shall use Corollary 5.1.1 to compute the expectation of the trace of the product of central functions $f_{1}$ and $f_{2}$ of these Wilson loops, obtaining

$$
\left\langle f_{1}(\alpha(p)) f_{2}(\beta(q))\right\rangle=\frac{1}{Z_{0}} \int f_{1}(x) f_{2}\left(y^{-1}\right) H\left(x y x^{-1} y^{-1} ; 2|M|\right) d x d y .
$$

For the expectation of any central function $f$ of such Wilson loops, we find

$$
\left\langle f\left(\alpha(p)^{-1} \beta(q)\right)\right\rangle=\langle f(\beta(q))\rangle=\left\langle f\left(\alpha^{-1}(p)\right)\right\rangle .
$$

If $p_{1} \leqq p_{2} \in a$ and $q_{1} \leqq q_{2} \in b$, we obtain

$$
\left\langle f\left(\alpha\left(p_{1}\right)^{-1} \alpha\left(p_{2}\right)\right)\right\rangle=\frac{1}{Z_{0}} \int f(\bar{x}) H\left(\bar{x} ; \Delta t_{1}\right) H\left(\bar{x}^{-1} x y x^{-1} y^{-1} ; 2|M|-\Delta t_{1}\right) d \bar{x} d x d y,
$$

and

$$
\begin{aligned}
& \left\langle f\left(\beta\left(q_{1}\right) \alpha\left(p_{1}\right) \beta\left(q_{2}\right)^{-1} \alpha\left(p_{2}\right)^{-1}\right)\right\rangle \\
& \quad=\frac{1}{Z_{0}} \int f(\bar{x}) H\left(\bar{x} ; \Delta t_{1}+\Delta t_{2}\right) H\left(\bar{x}^{-1} x y x^{-1} y^{-1} ; 2|M|-\left(\Delta t_{1}+\Delta t_{2}\right)\right) d \bar{x} d x d y,
\end{aligned}
$$

where the integrals are with respect to the Haar measure on $G, \Delta t_{1}=t_{1}\left(p_{2}\right)-t_{1}\left(p_{1}\right)$, $\Delta t_{2}=t_{2}\left(q_{2}\right)-t_{2}\left(q_{1}\right)$ and

$$
Z_{0}=\int H\left(x y x^{-1} y^{-1} ; 2|M|\right) d x d y .
$$

Remark 8.1. Equation (7.3) makes sense in the trivial cases $f=1$ and $p_{1}=p_{2}$. The convolution property of the heat kernel

$$
\int H\left(x_{1}^{-1} y ; t_{1}\right) H\left(y^{-1} x_{2} ; t_{2}\right) d y=H\left(x_{1}^{-1} x_{2} ; t_{1}+t_{2}\right)
$$

gives $\langle 1\rangle=1$ in the first case. In the second, the fact that $H(\bar{x} ; t)$ approaches a delta function of the identity as $t$ approaches zero gives $\langle f(\mathbf{1})\rangle=f(\mathbf{1})$.

Remark 8.2. Classically, $\alpha\left(p_{1}\right)=\alpha\left(p_{2}\right)$, since the paths in $M$ defining the holonomies are homotopic and the classical connection has zero curvature. As a consequence, $f(\alpha(p))$ would be indepenent of $p$. Equation (7.1) indicates that this aspect persists at the quantum level. However, the difference between the right-hand side of Eq. (7.3) and $f(\mathbf{1})$ is a quantum effect.

Remark 8.3. Equation (7.4) is the expectation of a function of holonomy about a contractible closed path. It differs from the corresponding result on $S^{2}$ only by the factor $x y x^{-1} y^{-1}$ and the integration over these variables. The generalization to higher genus seems clear, though we do not present it here.

To prove Eq. (7.3), begin with Corollary 5.1.1,

$$
\left\langle f\left(\alpha\left(p_{1}\right)^{-1} \alpha\left(p_{2}\right)\right)\right\rangle=\frac{1}{Z_{0}} \int_{\operatorname{Path}^{2} G} f\left(\alpha\left(p_{1}\right)^{-1} \alpha\left(p_{2}\right)\right) \exp \left[-\frac{1}{2} \sum_{i=1}^{2} \int_{0}^{T_{i}}\left|\frac{d \gamma_{i}}{d t_{i}}\right|^{2}\right] \mathscr{D} \alpha \mathscr{D} \beta .
$$

To compute the right-hand side, we interpret the path integration as Wiener measure. If the paths were constrained to take the endpoint values $\alpha(0)=x, \alpha(1)=y$, 
$\beta(0)=z$, and $\beta(1)=w$, then, as in [2], we could refer to Glimm and Jaffe [3] for the relation between the Wiener measure and the heat kernel. The expectation would be $\frac{1}{Z_{0}} \int H\left(x^{-1} \bar{x} ; t\left(p_{1}\right)\right) f\left(\bar{x}^{-1} \bar{y}\right) H\left(\bar{x}^{-1} \bar{y} ; t\left(p_{2}\right)-t\left(p_{1}\right) H\left(\bar{y}^{-1} y ; T_{1}-t\left(p_{2}\right)\right) H\left(z^{-1} w ; T_{2}\right) d \bar{x} d \bar{y}\right.$ and the normalization constant would be

$$
Z_{0}=H\left(x^{-1} y ; T_{1}\right) H\left(z^{-1} w ; T_{2}\right) .
$$

However, the only constraint is the endpoint relation of Theorem 3.1, which requires

$$
x w^{-1} y^{-1} z=1 \text {. }
$$

To relax to this weaker condition, we integrate the preceding expression over all possible values of $x, y$ and $z$, after setting $w=y^{-1} z x$. The expectation is then

$$
\begin{aligned}
\left\langle f\left(\alpha\left(p_{1}\right)^{-1} \alpha\left(p_{2}\right)\right)\right\rangle \\
=\frac{1}{Z_{0}} \int H\left(x^{-1} \bar{x} ; t\left(p_{1}\right)\right) f\left(\bar{x}^{-1} \bar{y}\right) H\left(\bar{x}^{-1} \bar{y} ; t\left(p_{2}\right)-t\left(p_{1}\right)\right) H\left(\bar{y}^{-1} y ; T_{1}-t\left(p_{2}\right)\right) \\
\quad \cdot H\left(z^{-1} y^{-1} z x ; T_{2}\right) d \bar{x} d \bar{y} d x d y d z,
\end{aligned}
$$

where

$$
Z_{0}=\int H\left(x^{-1} y ; T_{1}\right) H\left(z^{-1} y^{-1} z x ; T_{2}\right) d x d y d z .
$$

To evaluate the constant $Z_{0}$, use the convolution property of the heat kernel to integrate over $x$. Similarly, in Eq. (8.5), the convolution property gives the integral over $x$ directly. To proceed further, change variables from $\bar{y}$ to $\bar{x}^{-1} \bar{y}$, and use the invariance of the heat kernel under the adjoint action of $G$ on its first argument to integrate over $\bar{x}$. The proofs of the other equations in the corollary are similar.

We have computed this simple example quite explicitly. Computing similar expectations, for instance $\left\langle f\left(\prod \alpha_{i}\left(p_{j}\right)^{-1} \alpha_{i}\left(p_{j+1}\right)\right)\right\rangle$ on a surface of any genus, is more tedious but not fundamentally different. To give an indication of the role of the genus, we mention that for a surface of genus $g$, the partition function is

$$
Z_{0}=\int H\left(x_{1} y_{1} x_{1}^{-1} y_{1}^{-1} \cdots x_{g} y_{g} x_{g}^{-1} y_{g}^{-1} ; 2|M|\right) d x_{1} \cdots d y_{g} .
$$

The proof is entirely along the lines of the above calculation for $g=1$. Referring to Fegan [2] for the expansion of the heat kernel in terms of characters,

$$
H(x ; t)=\sum_{\mu} \chi_{\mu}(1) \chi_{\mu}(x) e^{-2 c(\mu) t},
$$

where $c(\mu)$ is the eigenvalue in the representation labelled by $\mu$ of the negative of the Laplacian on $G$, gives

$$
Z_{0}=\sum_{\mu} e^{-4|M| c(\mu)} \chi_{\mu}(1) \int \chi_{\mu}\left(x_{1} y_{1} x_{1}^{-1} y_{1}^{-1} \cdots x_{g} y_{g} x_{g}^{-1} y_{g}^{-1}\right) d x_{1} \cdots d y_{g}
$$

To compute the integral, use the convolution property of characters

$$
\int \chi_{\mu}(w x) \chi_{\nu}\left(x^{-1} z\right) d x=\delta_{\mu \nu} \frac{\chi_{\mu}(w z)}{\chi_{\mu}(\mathbf{1})}
$$


and the related formula (see [1, Chap. II, Sect. 4])

$$
\int \chi_{\mu}\left(w y z y^{-1}\right) d y=\frac{\chi_{\mu}(w) \chi_{\mu}(z)}{\chi_{\mu}(\mathbf{1})} \text {. }
$$

The integral over $2 g$ copies of $G$ becomes a factor $\chi_{\mu}(\mathbf{1})\left[\chi_{\mu}(\mathbf{1})\right]^{-2 g}$. The partition function is thus

$$
Z_{0}=\sum_{\mu} e^{-4|M| c(\mu)}\left[\chi_{\mu}(1)\right]^{2-2 g} .
$$

Witten [8] uses a lattice model to compute the same partition function combinatorially. His result is in close agreement with ours; the only difference is an overall factor of $\frac{1}{8}$ in the exponent. We could make the agreement exact by rescaling the volumes of Riemann surfaces of every genus simultaneously by a constant factor.

It might seem the above class of examples is severely limited, in that Wilson loops must be holonomy about radial paths. Recall, however, that we specified the fundamental domain $D$ only loosely. For any finite collection $S$ of noncontractible, non-self-intersecting closed paths on $M$ intersecting each other only at $m$, there is a choice of $D$ for which the paths in $S$ are radial. With this choice for $D$, the measure $\xi_{*} \mu$ defines the quantum expectations of functions of the Wilson loops determined by holonomy about elements of $S$. Thus, for example, Eq. (7.2) implies that the expectation of a function of holonomy about any non-contractible closed path is independent of the choice of path.

\section{Conclusion}

We have shown, for a Riemann surface of genus $g$, that $\mathscr{A} / \mathscr{G}_{m}$ is an affine linear bundle over Path ${ }^{2 g} G$. With a particular global section of this bundle defining a choice of origin on each fiber, the Yang-Mills measure on $\mathscr{A} / \mathscr{G}_{m}$ decomposes into that of a free theory on the fiber and, on the base, the product of Wiener measures on the component paths, conditioned on an endpoint relation. The areas enclosed by members of a 1-parameter family of closed paths on $M$ parametrize the paths in $\mathrm{Path}^{2 g} G$. Conformal changes of the metric on $M$ affect the measure on $\mathrm{Path}^{2 g} G$ only through these parametrizations. The methods and the results are a natural generalization of the theory for a bundle over $S^{2}$. By these methods, we have learned that a quantum theory of Yang-Mills in curved Euclidean space-time exists in two dimensions, for any genus, and we have shown how to compute some Wilson loop expectations in the theory. Interpreting the Yang-Mills path integral to mean an integral over $\mathscr{A} / \mathscr{G}_{m}$ with respect to the push-forward of a gauge-invariant measure on $\mathscr{A}$ was crucial in this approach.

Acknowledgements. I would like to express my gratitude to I. M. Singer for his comments and encouragement. I would also like to thank E. Witten who kindly sent me the preprint [8] in which I learned to the formula (8.7) which I had sought in vain.

\section{References}

1. Bröcker, T., tom Dieck, T.: Representations of Compact Lie Groups. Berlin, Heidelberg, New York: Springer 1985 
2. Fegan, H. D.: The Heat Equation on a Compact Lie Group. Trans. Am. Math. Soc. 246, 339-357 (1978)

3. Fine, D.: Quantum Yang-Mills on the Two-Sphere. Commun. Math. Phys. 134, 273-292 (1990)

4. Glimm, J., Jaffe, A.: Quantum Physics. New York, Berlin, Heidelberg: Springer 1987

5. Gross, L., King, C., Sengupta, A.: Two Dimensional Yang-Mills Theory via Stochastic Differential Equations. Ann. Phys. 194 (1), 65-112 (1989)

6. Sengupta, A.: The Yang-Mills Measure for the Two-Sphere. Ph.D. Dissertation Cornell University (1990)

7. Uhlenbeck, K. K.: Connections with $L^{p}$ Bounds on Curvature. Commun. Math. Phys. 83, 31-42 (1982)

8. Witten, E.: On Quantum Gauge Theories in Two Dimensions (preprint IASSNS-HEP-91/3, January 1991)

Communicated by A. Jaffe 